\title{
A (des)educação contraceptiva dos jovens universitários
}

\author{
Contraceptive (mis)education among young adults in Higher Education \\ La (des)educación contraceptiva de jóvenes universitarios
}

João Francisco de Castro ${ }^{1}$ io https://orcid.org/ 0000-0003-4090-9246

Carlos Manuel Torres Almeida ${ }^{1}$ io https://orcid.org/ 0000-0002-4497-4267

Vitor Manuel Costa Pereira Rodrigues ${ }^{1}$ io https://orid.org/ 0000-0002-2795-685X

Como citar:

Castro JF, Almeida CM, Rodrigues VM.

A (des)educação contraceptiva dos

jovens universitários. Acta Paul Enferm. 2020;33:APE201901306.

DOI

http://dx.doi.org/10.37689/actaape/2020A001916

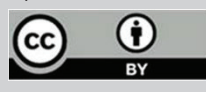

Descritores superior; Saúde sexua e reprodutiva; Portugal

Keywords Sexual education; Education, higher; Sexual and reproductive health ; Portugal

Descriptores

Educación sexual: Educación superior: Salud sexual y reproductiva, Portugal

\section{Submetido}

22 de Julho de 2019

Aceito

15 de Junho de 2020

\section{Autor correspondente \\ João Francisco de Castro \\ E-mail: jcastro@utad.pt}

\section{Resumo}

Objetivo: Investigar o impacto que o mais recente enquadramento legal da educação sexual teve na vivência da sexualidade de jovens universitários segundo comportamentos protetores, profiláticos e de vigilância em saúde sexual e reprodutiva.

Métodos: Trata-se de um estudo de natureza quantitativa, transversal e descritivo, com uma amostra não probabilística de conveniência, constituída por 365 universitários do norte de Portugal. Foi critério de inclusão os estudantes frequentarem $01^{\circ}$ ano de licenciatura, procurando recrutar estudantes mais jovens, que já foram abrangidos em todo 0 Ensino Secundário pela Lei 60/2009 de 06 de agosto, relativa à educação sexual em meio escolar. Utilizámos um questionário na coleta de dados, que analisámos recorrendo à estatística descritiva e inferencial com um nível de confiança de 95\%.

Resultados: A procura pelos serviços de saúde, no âmbito da saúde sexual e reprodutiva é baixa, apenas $28,7 \%$ frequenta as consultas de planejamento familiar, sendo o sexo feminino quem mais reporta este fato $(\chi 2=41,085$ e $p<0,000)$; o recurso aos serviços de saúde da universidade é ainda menor $(13,7 \%)$, também a procura é maior pelo sexo feminino em relação masculino (11,2\% versus $2,5 \%)$. Dos estudantes sexualmente ativos, 29,6\% não faz uma utilização sistemática de métodos contraceptivos, estando este comportamento mais presente no sexo masculino $(\chi 2=9,950$ e $p=0,002)$.

Conclusão: Comportamentos protetores no âmbito da sexualidade estão pouco presentes, devendo a intervenção junto dos jovens ultrapassar a genitalidade para uma visão holística e integradora da sexualidade.

\section{Abstract}

Objective: To investigate how the current law on sex education has affected the sex life of young adults in Higher Education on the basis of protective, prophylactic, and sexual and reproductive health surveillance behaviors.

Method: This was a quantitative cross-sectional descriptive study with a non-probability sample of convenience consisting of 365 Higher Education students from Northern Portugal. We included students attending the first year of Teaching Degree programs, therefore, younger students who would have had their Secondary Education under Law no. 60/2009 (August 6) would be included (this Law currently regulates sex education in schools). Data were collected using a questionnaire, which was submitted to descriptive and inferential statistical analysis with a confidence level of 95\%.

Results: For sexual and reproductive health, health services are rarely sought: only $28.7 \%$ of respondents have participate in family planning appointments, and women reported seek it more often $(\chi 2=41.085$ and $p<0.000)$ than men. The university's health services are even more rarely sought $(13.7 \%)$, and women also

'Centro de Investigação em Desporto, Saúde e Desenvolvimento Humano, Escola Superior de Saúde, Universidade de Trás-os-Montes e Alto Douro, Vila Real, Portugal.

Conflitos de interesse: nada a declarar. 
seek it more often than men (11.2\% versus $2.5 \%$ ). Of participants, $29.6 \%$ of sexually active students do not systematically use contraceptive methods, and this behavior is more prevalent among men $(\chi 2=9.950$ e $p=0.002)$.

Conclusion: Protective sexual behaviors are rarely present. The study recommends any interventions targeting young adults that should go beyond genital anatomy and need to introduce a holistic and integrating viewing of sexuality.

\section{Resumen}

Objetivo: Investigar el impacto del más reciente marco legal de la educación sexual en la vivencia de la sexualidad de jóvenes universitarios según comportamientos de protección, de profilaxis y de control de la salud sexual y reproductiva.

Métodos: Se trata de un estudio de naturaleza cuantitativa, transversal y descriptiva, con muestreo no probabilístico por conveniencia, constituido por 365 universitarios del norte de Portugal. Fue un criterio de inclusión que los estudiantes estuvieran cursando $1^{\circ}$ año de licenciatura, para reclutar estudiantes más jóvenes que hayan pasado toda la enseñanza secundaria regidos por la Ley 60/2009 del 06 de agosto, relacionada con la educación sexual en medio escolar. En la recolección de datos utilizamos un cuestionario que analizamos a través de la estadística descriptiva e inferencial, con un nivel de confianza del 95 \%.

Resultados: En el ámbito de la salud sexual y reproductiva, la demanda de servicios de salud es baja, solo el 28,7 \% asiste a consultas de planificación familiar, y quien más reporta este hecho es el sexo femenino $(\chi 2=41,085$ y $p<0,000)$. Este porcentaje es aún menor en quienes recurren a los servicios de salud de la universidad (13,7\%), la demanda también es mayor en el sexo femenino con relación al masculino (11,2\% versus 2,5\%). De los estudiantes sexualmente activos, el 29,6 \% no utiliza métodos contraceptivos de forma sistemática, y este comportamiento está más presente en el sexo masculino ( $\chi 2=9,950$ y p=0,002).

Conclusión: Los comportamientos de protección en el ámbito de la sexualidad están poco presentes, y la intervención con los jóvenes debe ir más allá de la genitalidad, hacia una visión holística e integradora de la sexualidad.

\section{Introdução}

Atualmente multiplicam-se os estudos no âmbito da saúde sexual e reprodutiva (SSR), pelo seu impacto no bem-estar geral da sociedade. O grupo dos jovens tem sido particularmente visado, pois tem-se assistido a uma iniciação sexual cada vez mais precoce o que no contexto da imaturidade dos jovens se traduz muitas vezes na adoção de comportamentos de risco. Estes são particularmente evidentes pelo não uso ou uso inadequado dos métodos contraceptivos, o que expõe os jovens às infecções sexualmente transmissíveis (IST's) e à gravidez indesejada. ${ }^{(1,2)}$

A educação sexual dos jovens constitui a mais importante forma de prevenção de problemas ligados à SSR, podendo contribuir para ajudar os adolescentes a tomar decisôes mais adequadas nesta área, pois o fato de terem atualmente muita facilidade em obter informação, não garante que as suas escolhas sejam as mais adequadas. ${ }^{(2,3)}$

Esta educação sexual passa em primeiro lugar pelos pais e família, mas o fato é que esta se constitui como um processo contínuo e multiparticipado, com o crescimento e socialização da criança há toda uma série de agentes que intervêm direta ou indiretamente nesta aprendizagem de temas relacionados com a sexualidade. ${ }^{(1,2)}$

Entre nós só depois da queda de uma ditadura política em Abril de 1974, foi publicada a primeira lei de educação sexual em 24 de março de 1984 . A
Lei no 3/84 que versava sobre a "Educação sexual e planejamento familiar", e que logo no seu segundo artigo se debruçava sobre a educação sexual dos jovens, que devia ser garantida pelo estado através da escola, organizaçóes sanitárias e dos meios de comunicação social, em cooperação com os pais. ${ }^{(2)}$

Seguiu-se toda uma evolução legislativa que visa progressivamente assegurar a efetividade desta incumbência do estado, nomeadamente a garantia do direito à SSR, apelando sempre à participação dos pais e encarregados de educação. Atualmente é a Lei no 60/2009 de 06 de agosto que rege a educação sexual em meio escolar e que abrange todos os ciclos do ensino básico e também o ensino secundário, ficando apenas de fora o ensino pré-escolar e o ensino superior. ${ }^{(1,2)}$

A regulamentação da Lei no 60/2009, efetuada através da Portaria no 196-A/2010 de 09 de abril, objetiva claramente os conteúdos mais centrados nos aspetos biológicos e anatómicos que se prendem com objetivos utilitários, como a redução das IST's e da gravidez indesejada, que são importantes mas no entanto redutores para representarem uma concepção global da sexualidade. Já na educação para os valores ou princípios éticos para uma vivência da sexualidade, limita-se à expressão generalista "Aspectos éticos da sexualidade", não acrescentando qualquer outra especificação.

Os dados do relatório dos comportamentos de saúde dos jovens universitários portugueses, com dados nacionais de 2016, ${ }^{(4)}$ parecem colocar em causa a 
efetividade deste normativo legal, nomeadamente no que diz respeito á diminuição dos comportamentos de risco. Verificou-se, entre os estudantes que já tiveram relaçôes sexuais, um aumento de 2010 para 2016 dos que mencionaram ter engravidado sem desejar $(4,1 \%$ para $6 \%$ ), ter tido uma infecção sexualmente transmissível (3,3\% para 5,2\%), e ter realizado uma interrupção voluntária da gravidez $(3,2 \%$ para $4,5 \%) .{ }^{(4)}$

A passagem dos jovens do ensino secundário para o ensino universitário representa mais um passo na sua progressiva autonomia, ${ }^{(3,4)}$ esta maior autonomia em relação à família, sobretudo nos estudantes deslocados, implica também uma maior responsabilidade nas suas opçôes de vida, verificando-se muitas vezes o questionar de convicçóes, crenças e valores por estes jovens adultos levando-os a adotar comportamentos pouco saudáveis. ${ }^{(5,6)}$

Estes comportamentos prendem-se com o consumo de substâncias e práticas sexuais condizentes a oportunidades e práticas de risco, nomeadamente o uso inconsistente de métodos contraceptivos e do preservativo, a existência de parceiros ocasionais e a associação entre o consumo de álcool e/ou drogas e comportamentos sexuais. ${ }^{(5,7-9)}$ A prevalência destes riscos tem vindo a aumentar em particular nos jovens adultos, ${ }^{(4)}$ constituindo um importante problema de saúde pública que tem sido alvo de vários trabalhos de investigação, que procuram compreender os fatores subjacentes à adoção de comportamentos de risco pelos jovens, constituindo um grupo social prioritário de intervenção. ${ }^{(7)}$

Sabendo que por si só o acesso à informação não garante as escolhas mais adequadas ao nível da SSR, ${ }^{(2)}$ e reconhecido o valor que a educação sexual pode ter neste aspecto; quisemos averiguar o impacto que o mais recente enquadramento legal da educação sexual teve na adoção de comportamentos protetores na vivência da sexualidade, nomeadamente pela existência de comportamentos de risco e existência de comportamentos profiláticos ou de vigilância em SSR.

\section{Métodos}

Tendo em conta os objetivos traçados, desenvolvemos um estudo de natureza quantitativa, transver- sal e descritivo numa amostra de estudantes de uma Universidade do norte de Portugal. Recorremos para o efeito a uma amostra não probabilística de conveniência, constituída por 365 estudantes, todos a frequentar o $1^{\circ}$ ano dos cursos de licenciatura dessa Universidade. Como critérios de exclusão foram utilizados os seguintes: estudantes do $2^{\circ}, 3^{\circ}$ e $4^{\circ}$ anos de licenciatura e estudantes de mestrado e doutoramento. Procuramos assim recrutar estudantes que já foram abrangidos em todo o ensino secundário pela Lei no 60/2009 de 06 de agosto, relativa à educação sexual em meio escolar.

A coleta de dados foi realizada, durante o primeiro semestre de 2014, através de um questionário de autopreenchimento, construído especificamente para o efeito, sendo constituído por 41 perguntas abertas e fechadas com um tempo médio de preenchimento de 13 minutos. Foi realizado um pré-teste com 12 estudantes que não revelou dificuldades no seu preenchimento. O questionário depois de aprovado pela Comissão de Ética (Parecer no 07/2014) foi aplicado em sala de aula, após explicação aos estudantes dos objetivos do estudo e esclarecidos sobre o caráter anônimo e participação voluntária. $\mathrm{O}$ questionário, permitiu recolher dados sociodemográficos, acadêmicos, de comportamentos sexuais bem como as práticas de vigilância em SSR.

O tratamento estatístico dos dados, foi efetuado através do programa Statistic Package for the Social Sciences (SPSS) na versão 25.0, que analisámos recorrendo à estatística descritiva e inferencial, com um nível de confiança de $95 \%$.

\section{Resultados}

$\mathrm{Na}$ amostra constituída por 365 estudantes, verificámos que 254 são meninas $(69,6 \%)$, a média de idades é de 19 anos $(19,46+2,79)$, sendo a mediana e a moda de 19 e 18 anos respectivamente. Quase metade dos estudantes é proveniente da cidade (46,8\%), solteira $(95,9 \%)$ e católica $(83,8 \%)$. As famílias têm baixa escolaridade, $50,4 \%$ das mães e $61,6 \%$ dos pais possuem apenas o ensino básico ( $9^{\circ}$ ano ou inferior).

Apenas 33,4\% dos estudantes afirma falar com os pais de sexualidade/contracepção, sendo que este fato 
é mais reportado pelo sexo feminino, com diferenças estatisticamente significativas $(\chi 2=6,741$ e $\mathrm{p}=0,034)$. Entre aqueles que falam às vezes ou não falam a razão mais referida foi "tenho vergonha/receio" $(15,1 \%)$.

Praticamente todos os estudantes (96,4\%), referem ter tido aulas em que foi abordada a temática da sexualidade/contracepção. No entanto quando questionados onde adquiriram essencialmente os conhecimentos sobre sexualidade/contracepção, em primeiro lugar são referidos os "profissionais de saúde" (61,9\%), depois os "amigos e colegas" (54,8\%) e só depois os "professores" (47,9\%). Esta informação junto dos profissionais de saúde é mais referida pelo sexo feminino, com diferenças estatisticamente significativas em relação ao sexo masculino $(\chi 2=6,320$ e p=0,012).

$\mathrm{Na}$ tabela 1 , podemos observar que a maioria dos estudantes (69,3\%) já teve relaçôes sexuais. Tendo em conta o grupo sexual, este fato é mais referido pelo sexo masculino, tendo as diferenças significado estatístico. Na primeira relação sexual, 97,2\% diz ter utilizado ou o parceiro/a algum método contraceptivo, sendo o preservativo o mais referido (74\%) e depois a combinação da pílula mais preservativo (29,3\%). A idade média da primeira relação sexual foram os 17 anos $(16,72 \pm 1,51)$, tendo em conta o grupo sexual a idade média é menor no sexo masculino em relação ao feminino $(16,32$ versus 16,93), sendo que esta diferença de médias tem significado estatístico $(\mathrm{t}=-3,073$ e $\mathrm{p}=0,002)$.

No que respeita a comportamentos de risco, verificamos que quase $30 \%$ dos estudantes não faz uma utilização sistemática de métodos contraceptivos, sendo este comportamento significativamente mais referido pelo sexo masculino. Nestes, a razão mais destacada foi "por escolha deliberada" (67,7\%), seguindo-se "por interferir com o relacionamento sexual" (29\%). Dos estudantes que referem ter utilizado algum método contraceptivo, como ultimo método utilizado o mais referido foi o preservativo (46\%) seguindo-se a pílula (34,9\%). A maioria dos estudantes refere que não teve relaçóes sexuais sob o efeito do álcool e relaçóes sexuais ocasionais, porém nestas duas situaçóes há sempre respostas com uma percentagem superior a 33\% (Tabela 1), que referem esses comportamentos, ou seja pelo menos 1 em cada 3 estudantes. É o sexo masculino quem mais apresenta estes comportamentos, tendo as diferenças para o sexo feminino, significado estatístico.
Tabela 1. 0corrência de relações sexuais e comportamentos de risco no âmbito da saúde sexual e reprodutiva

\begin{tabular}{|c|c|c|c|c|}
\hline Variáveis & $\begin{array}{l}\text { Masculino } \\
\mathrm{n}(\%)\end{array}$ & $\begin{array}{c}\text { Feminino } \\
\mathrm{n}(\%)\end{array}$ & $\begin{array}{l}\text { Total } \\
\mathrm{n}(\%)\end{array}$ & $\chi^{2}$ \\
\hline \multicolumn{5}{|c|}{$\begin{array}{l}\text { Alguma vez teve relações sexuais } \\
(n=365)\end{array}$} \\
\hline Sim & $88(24,1)$ & $165(45,2)$ & $253(69,3)$ & $7,446^{\star \star}$ \\
\hline Não & $23(6,3)$ & $89(24,4)$ & $112(30,7)$ & \\
\hline \multicolumn{5}{|c|}{$\begin{array}{l}\text { Utilização sistemática de métodos } \\
\text { contraceptivos }(n=253)\end{array}$} \\
\hline Sim & $51(20,2)$ & $127(50,2)$ & $178(70,4)$ & $9,950^{\star \star}$ \\
\hline Não & $37(14,6)$ & $38(15)$ & $75(29,6)$ & \\
\hline \multicolumn{5}{|c|}{$\begin{array}{l}\text { Alguma vez teve relações sexuais sob } \\
0 \text { efeito de drogas ilícitas }(n=251)\end{array}$} \\
\hline Sim & $20(8)$ & $22(8,8)$ & $42(16,7)$ & 3,494 \\
\hline Não & $68(27,1)$ & $141(56,2)$ & $209(83,3)$ & \\
\hline \multicolumn{5}{|c|}{$\begin{array}{l}\text { Alguma vez teve relações sexuais } \\
\text { sob o efeito do consumo de álcool } \\
(n=251)\end{array}$} \\
\hline Sim & $52(20,7)$ & $43(17,1)$ & $95(37,8)$ & $27,201^{\star \star \star}$ \\
\hline Não & $35(13,9)$ & $121(48,2)$ & $156(62,2)$ & \\
\hline \multicolumn{5}{|c|}{$\begin{array}{l}\text { Teve relações sexuais ocasionais ( } \mathrm{n} \\
=251 \text { ) }\end{array}$} \\
\hline Sim & $54(21,5)$ & $29(11,6)$ & $83(33,1)$ & $50,599^{\star \star \star}$ \\
\hline Não & $33(13,1)$ & $135(53,8)$ & $168(66,9)$ & \\
\hline
\end{tabular}

${ }^{*} \mathrm{p} \leq 0,05 ;{ }^{* \star} \mathrm{p} \leq 0,01 ;{ }^{* \star \star} \mathrm{p} \leq 0,001$

O recurso à contracepção de emergência, teve uma prevalência de 19,7\% na amostra de estudantes. As razóes mais referidas para este recurso foram "falha do método contraceptivo" (64,3\%) e "relação sexual desprotegida" (42,9\%).

Relativamente à vigilância em SSR (Tabela 2), $71,3 \%$ diz não frequentar a consulta de planejamento familiar, e uma percentagem ainda maior $(86,3 \%)$ diz não recorrer aos serviços de saúde da instituição no âmbito da SSR. O recurso a estes serviços é mais referido pelo sexo feminino, tendo as diferenças para o sexo masculino, significado estatístico. Já na realização do teste ao vírus da imunodeficiência humana (VIH), é o sexo masculino quem mais o refere com diferenças significativas em relação ao sexo feminino.

Questionados os estudantes acerca de quando é mais fácil uma menina ficar grávida, é paradigmático que pouco mais de metade dos estudantes $(54,8 \%)$, apontou o meio do ciclo como a altura mais provável. Analisando só respostas das meninas, pudemos verificar que $62,9 \%$ não faz auto-exame da mama, 77,4\% náo faz citologia cervicovaginal (Papanicolau), 75,1\% fez vacinação contra o Papiloma Vírus Humano (HPV) o que equivale a dizer que praticamente 1 em cada 4 não fez essa vacinação. Das meninas 62,5\% faz contracepção oral, mas destas 55,5\% não faz a toma certa da pílula em 
Tabela 2. Vigilância em saúde sexual e reprodutiva

\begin{tabular}{|c|c|c|c|c|}
\hline Variáveis & $\begin{array}{c}\text { Masculino } \\
\mathrm{n}(\%)\end{array}$ & $\begin{array}{c}\text { Feminino } \\
\mathrm{n}(\%)\end{array}$ & $\begin{array}{l}\text { Total } \\
\mathrm{n}(\%)\end{array}$ & $\chi^{2}$ \\
\hline \multicolumn{5}{|c|}{$\begin{array}{l}\text { Frequenta a consulta de planejamento } \\
\text { familiar }(n=362)\end{array}$} \\
\hline $\operatorname{Sim}$ & $6(1,7)$ & $98(27,1)$ & $104(28,7)$ & \multirow{2}{*}{$41,085^{\star \star \star}$} \\
\hline Não & $103(28,5)$ & $155(42,8)$ & $258(71,3)$ & \\
\hline \multicolumn{5}{|c|}{$\begin{array}{l}\text { Já recorreu no âmbito da SSR aos } \\
\text { serviços de saúde da sua instituição } \\
(\mathrm{n}=365))\end{array}$} \\
\hline $\operatorname{Sim}$ & $9(2,5)$ & $41(11,2)$ & $50(13,7)$ & \multirow{2}{*}{$4,217^{\star}$} \\
\hline Não & $102(27,9)$ & $213(58,4)$ & $315(86,3)$ & \\
\hline \multicolumn{5}{|c|}{ Já fez o teste do VIH $(n=363)$} \\
\hline Sim & $24(6,6)$ & $25(6,9)$ & $49(13,5)$ & \multirow{2}{*}{$9,355^{\star \star}$} \\
\hline Não & $86(23,7)$ & $228(62,8)$ & $314(86,5)$ & \\
\hline
\end{tabular}

todos os ciclos; das que se esquecem de tomar a pílula quando questionadas acerca do que fazem nessa ocasião, 55,3\% "nada, continua a toma", 27,1\% "toma duas" e apenas 17,6\% afirma "usa outro método". No entanto a maioria considera globalmente os seus conhecimentos sobre sexualidade/contracepção, como suficientes $(62,7 \%)$ ou bons $(34,2 \%)$.

\section{Discussão}

Constata-se que os estudantes que participaram neste estudo, apresentam resultados similares a outros estudos nacionais nomeadamente em relação ao sexo predominante, jovens sexualmente ativos e idade da primeira relação sexual. ${ }^{(1,4,10)}$ Apesar de os nossos dados contemplarem apenas estudantes do $1^{\circ}$ ano da licenciatura, os valores dos jovens sexualmente ativos e da idade da primeira relação sexual, estão muito próximos dos dados nacionais de $2016^{(4)}$ e são praticamente sobreponíveis aos de um estudo nacional que também só abrangeu alunos do $1^{\mathrm{o}}$ ano da licenciatura. ${ }^{(10)}$ No entanto, pelo fato apontado a idade média dos participantes é um pouco inferior a outros estudos nacionais. ${ }^{(1,11)}$

Registramos igualmente que são maioritariamente os jovens do sexo masculino e que mais precocemente, iniciam uma vida sexual ativa, ${ }^{(1,3)}$ esta tendência para a iniciação sexual é não só fruto da pressão dos colegas mas também das maravilhas associadas que os mass media parecem transmitir. ${ }^{(1)}$ Algumas concepçóes de género de índole tradicional podem explicar, que enquanto entre as jovens parece persistir a ideia romântica da entrega ao parceiro através da primeira relação sexual sendo concedida a própria virgindade, entre os rapazes a sexualidade e sentimento são duas realidades distintas que podem eventualmente sobrepor-se, e a primeira relação sexual é vista como uma prova de iniciação um desafio que ele deve superar para provar a sua competência sexual. ${ }^{(12,13)}$

O início precoce da atividade sexual representa um factor de risco para a adoção de comportamentos de risco, pois os adolescentes e jovens adultos estão mais propensos a correr riscos ${ }^{(1,5,14)}$ e por outro lado fruto da sua pouca experiência de vida, o conhecimento sobre métodos profiláticos é menor e o uso incorreto está mais presente. ${ }^{(5,15)}$ Reflexos disso são os dados nacionais de 2014 em que se reporta uma diminuição do uso do preservativo e aumento de relaçóes sexuais associadas ao consumo de drogas e álcool, sendo que aqueles que não utilizaram preservativo na $1^{\mathrm{a}}$ relação sexual apontaram como motivos, em primeiro lugar "não pensaram nisso" (42\%) e em segundo o "não tinha preservativos consigo" $(31,8 \%)$, ${ }^{(5)}$ o que remete nestes jovens para uma iniciaçáo sexual de risco e impregnada de irresponsabilidade, em que está evidente o caráter espontâneo e não planejado dos relacionamentos sexuais entre os jovens, o que leva a que a maioria se inicie sexualmente de uma forma imprevista. ${ }^{(1,5)}$

Apesar de a maioria ter referido a abordagem de conteúdos de sexualidade/contracepção em sala de aulas, ${ }^{(10,14)}$ os estudantes se considerarem bem infor$\operatorname{mados}^{(13)}$ e até referirem a utilização de métodos contraceptivos na primeira relação sexual, ${ }^{(4,10,13)}$ não deixam depois de apresentar comportamentos sexuais de risco significativos, como os verificados no presente estudo pela não utilizaçáo sistemática de métodos contraceptivos, ${ }^{(1,4,11)}$ relaçôes sexuais ocasionais ${ }^{(10,16,17)}$ e sob o efeito do consumo de álcool. ${ }^{(5,18)}$

Também um estudo nacional, com grupo de intervenção e avaliação aos 36 meses, demonstrou que alguns resultados importantes, nomeadamente a existência de relaçóes sexuais sob efeito do álcool e não uso de preservativo com parceiro ocasional, não só não melhoraram como sofreram um agravamento. ${ }^{(10)}$ Muitos destes comportamentos de risco acabam depois por motivar o recurso à contracepção de emergência, que no nosso estudo apresentou uma prevalência de $19,7 \%$, inferior a outros estudos, ${ }^{(11,13)}$ porventura por ser uma população "mais jovem". 
O que é um fato preocupante, é que apesar do atual enquadramento legal relativo à educação sexual em meio escolar, com horas previstas no currículo exclusivamente para esse efeito, se verificou um agravamento dos comportamentos sexuais de risco dos jovens universitários de 2010 para 2016 conforme dados nacionais, ${ }^{(4)}$ apesar da complementaridade de agentes prevista na lei para esta educação sexual, pois reconhece-se que nem sempre os pais estão suficientemente habilitados para esta, ${ }^{(11,19)}$ e os próprios estudantes mencionam outras fontes de informação. ${ }^{(10,11)}$ Entre estas encontram-se muitas vezes os profissionais de saúde ${ }^{(11)}$ mas muitos dos jovens não sentem necessidade de procurar os serviços de saúde como verificamos, para obter uma informação credível e completa, além do acesso gratuito aos diferentes métodos contraceptivos, persistindo um uso incorreto dos mesmos. ${ }^{(12,20)}$

Assim, num estudo nacional para avaliação das práticas contraceptivas das mulheres em Portugal, verificou-se precisamente que são as mulheres mais jovens que menos frequentam as consultas de planejamento familiar e em que o médico é menos referido como fonte de informação. ${ }^{(20)}$ Salientamos o fato de nos jovens do nosso estudo, que não fizeram uma utilização sistemática de métodos contraceptivos, a razão mais referida foi "por escolha deliberada" (67,7\%).

Estas primeiras escolhas que os jovens universitários fazem de forma mais autónoma, mas eventualmente menos ponderada, pode influenciar decisivamente a sua SSR. ${ }^{(3)}$ Mesmo entre as meninas, que tradicionalmente recorrem mais às consultas de planejamento familiar, verificamos uma percentagem reduzida na adesão a estas consultas $(27,1 \%)$ com efeitos potencialmente negativos, pelo não esclarecimento com os profissionais de saúde; ${ }^{(11,14)}$ e consequentemente a não implementação de medidas protetoras/profiláticas em relação à sua SSR (adequadas ao seu caso concreto), como sejam a escolha e utilização correta do método contraceptivo ou mesmo a realização regular da citologia cervico-vaginal.

Como a adesão à contracepção é importante no âmbito da SSR, compreender as razóes subjacentes à decisão, podem revelar a percepçáo da (in)vulnerabilidade ao risco. ${ }^{(1)}$ Coloca-se a questão, se com os avanços tecnológicos no combate às IST's, nomeadamente ao VIH e à síndrome da imunodeficiência adquirida (SIDA) com a introdução dos antirretrovirais, mudou a representação do risco e a sua percepçáo; ${ }^{(21)}$ e por outro lado se uma atitude mais favorável a uma contracepção de emergência (de venda livre entre nós), pode fomentar o relaxamento de condutas preventivas em relação à gravidez e IST`s. Ao transmitir uma sensação de segurança pela existência de um método anticonceptivo pós-coito, os comportamentos de risco podem-se multiplicar. ${ }^{(22)}$

Compreender os comportamentos sexuais para além do aporte cognitivo, ${ }^{(3,13,23)}$ torna-se essencial para a promoção da efetividade da educação sexual. A prevenção em SSR é fundamental e atitudes de negação do risco são das principais barreiras para a sua implementação. ${ }^{(24)}$

De referir como limitação ao estudo, o fato de se tratar de uma realidade local de Portugal enquadrada por politicas educativas próprias em matéria de educação sexual. Este é um assunto importante e emergente na sociedade contemporânea gerando preocupação constante, em praticamente todo o mundo, traduzindo-se num investimento crescente na educação sexual dos jovens tendo em vista a adoção de comportamentos protetores na vivência da sexualidade. Era desejável que o estudo que esteve na base do presente artigo, pudesse ser replicado em vários paises dos diferentes continentes, para sabermos a procura pelos serviços de saúde, no âmbito da SSR, se há pelos jovens, uma utilização sistemática de métodos contraceptivos e qual a existência de comportamentos protetores no âmbito da sexualidade.

\section{Conclusão}

Existe um grande número de jovens que iniciou uma vida sexual ativa onde os comportamentos de risco estão muito presentes, pelo não uso dos métodos contraceptivos ou pelo seu uso inconsistente, expondo os jovens estudantes a IST's e/ou gravidez indesejada. De referir que os jovens do sexo masculino, apresentam mais comportamentos de risco, nomeadamente no que se refere ás relaçôes sexuais sob o efeito do álcool, parceiros ocasionais e uso não sistemático de métodos contraceptivos. Os comportamentos protetores e/ou profiláticos no âmbito da SSR, são pouco referencia- 
dos pelos jovens nomeadamente o recurso às consultas de planejamento familiar e aos serviços de saúde da sua Instituição, no entanto estes estão mais presentes no sexo feminino. Os estudantes revelam pouca sensibilidade para esta área da promoção da SSR e recurso aos serviços existentes, dado que a sua procura ainda é limitada e os estudantes parecem não reconhecer a importância de uma vigilância de saúde regular no âmbito da SSR. Parece evidente como reconhecido em diferentes estudos que o conhecimento por si só não garante as escolhas mais adequadas, devendo a educação sexual ultrapassar um cunho muito genitalista e passar para uma visão mais holística e integradora da sexualidade e em que os aspetos psicossociais sejam amplamente explorados, afastando formatos standard de educação sexual e espartilhos ideológicos.

\section{Colaborações}

Castro JF, Almeida CMT e Rodrigues VMCP contribuíram com a concepção do estudo, análise e interpretação dos dados, redação do artigo, revisão crítica relevante do conteúdo intelectual e aprovação da versão final a ser publicada.

\section{Referências}

1. Pimentel $\mathrm{MH}$, Preto LS, Alves MJ, Monteiro AM. Comportamento sexual e estudantes do ensino superior. Psicol Saude Doencas. 2016;17(3):352-67.

2. Matos MG, Reis M, Ramiro L, Ribeiro JP, Leal I. Educação sexual em Portugal: legislação e avaliação da implementação nas escolas. Psicol Saude Doencas. 2014;15(2):35-55.

3. Sales WB, Caveião C, Visentin A, Mocelin D, Costa PM, Simm EB. Comportamento sexual de risco e conhecimento sobre IST/SIDA em universitários da saúde. Rev Enferm Ref . 2016; IV Serie(10):19-27.

4. Reis M, Matos MG. Equipa Aventura Social. Comportamentos de saúde dos jovens universitários portugueses: relatório do estudo - dados Nacionais de 2016. [Internet]. 2017 [citado 2019 Mai 03]. Disponível em: http://aventurasocial.com/arquivo/1499021788_JUNP_Relatorio_ junho2017_v8F_28junh02017.pdf

5. Matos MG, Simões C, Camacho I, Reis M. EquipaAventura Social. A saúde dos adolescentes portugueses em tempos de recessão: dados nacionais 2014. [Internet]. 2015 [citado 2019 Mai 3]. Disponível em: http://aventurasocial. com/arquivo/1437158618_RELATORI0\%20HBSC\%202014e.pdf

6. Foster C, Caravelis C, Kopak A. National College Health Assessment Measuring Negative Alcohol - Related Consequences among College Students. Am J Public Health Res. 2014;2(1):1-5.
7. World Health Organization (WHO). Adolescents: health risks and solutions. Fact sheet № 345 [Internet]. Genève; WHO; 2014 [cited 2019 May 3]. Available from: http://www.searo.who.int/thailand/ factsheets/fs0027/en/

8. Castro A. Sexual behavior and sexual risks among Spanish University Students: a descriptive study of gender and sexual orientation. Sex Res Soc Policy. 2016;13(1):84-94.

9. Parks KA, Frone MR, Muraven M, Boyd C. Nonmedical use of prescription drugs and related negative sexual events: prevalence estimates and correlates in college students. Addict Behav. 2017;65:258-63.

10. Oliveira AC, Caramelo F, Patrício M, Camarneiro AP, Cardoso SM, Pita JR. Impacto de um programa de intervenção educativa nos comportamentos sexuais de jovens universitários Rev Enferm Ref. 2017; IV Serie;(13):71-82.

11. Santos MJ, Ferreira EM, Ferreira MM. Contraceptive behavior of Portuguese higher education students. Rev Bras Enferm. 2018;71 Suppl 4:1706-13.

12. Delatorre M, Dias AC. Conhecimentos e práticas sobre métodos contraceptivos em estudantes universitários. Rev SPAGESP. 2015;16(1):60-73.

13. Nelas $P$, Chaves $C$, Coutinho E, Amaral 0 . Values and beliefs about sexuality, motherhood and abortion. International J Develop Educational Psychology. 2016;1(2):211-9.

14. Neto S, Bombas T, Arriaga C, Almeida MC, Moleiro P. Contraceção na adolescência: recomendações para 0 aconselhamento contracetivo. Acta Pediatr Port. 2014;45:51-63.

15. Santos MJ,FigueiredoA. Contraceção na adolescência: aconselhamento e linhas de orientação. Revi Eletrón Educ Psicol. 2015;4:62-75.

16. Mehra D, Kyagaba E, Ostergren PO, Agardh A. Association between self-reported academic performance and risky sexual behavior among Ugandan university students- a cross sectional study. Glob J Health Sci. 2014;6(4):183-95.

17. Hedayati-Moghaddam MR, Eftekharzadeh-Mashhadi I, Fathimoghadam F, Pourafzali SJ. Sexual and reproductive behaviors among undergraduate university students in Mashhad, a city in Northeast of Iran. J Reprod Infertil. 2015;16(1):43-8.

18. Rocha YA, Silva MA. Conhecimento sobre HIV/AIDS e práticas sexuais de estudantes de graduação em enfermagem. Estud Vida Saúde. 2014;41(2):275-89

19. Dantas KT, Spínola T, Teixeira SV, Lemos AC, Ferreira LE. Jovens universitários e o conhecimento acerca das doenças sexualmente transmissíveis - contribuição para cuidar em enfermagem. Rev Pesqu Cuid Fundametal Online. 2015;7(3):3020-36.

20. Águas F, Bombas T, Silva DP. Avaliação das práticas contracetivas das mulheres em Portugal. Portugal. Acta Obstet Ginecol Port. 2016;10(3):1-9.

21. Beltzer N, Saboni L, Sauvage C, Lydié N, Semaille C, Warszawski J; group KABP France. An 18-year follow-up of HIV knowledge, risk perception, and practices in young adults. AIDS. 2013;27(6):1011-9.

22. Brandão ER, Cabral CS, Ventura M, Paiva SP, Bastos LL, Oliveira NV, et al. "Bomba hormonal": os riscos da contracepção de emergência na perspectiva dos balconistas de farmácias no Rio de Janeiro, Brasil. Cad Saude Publica. 2016;32(9):e00136615.

23. Uecker JE. Social context and sexual intercourse among first-year students at selective colleges and universities in the United States. Soc Sci Res. 2015;52:59-71.

24. Deblonde J, Hamers FF, Callens S, Lucas R, Barros H, Rüütel $K$, et al. HIV testing practices as reported by HIV-infected patients in four European countries. AIDS Care. 2014;26(4):487-96. 